\title{
Schwangerschaftsassoziierte Thrombosen und Lungenembolien
}

Elgendy I et al. Clinical Characteristics and Outcomes of Women Presenting with Venous Thromboembolism during Pregnancy and Postpartum Period: Findings from the RIETE Registry. Thromb Haemost 2020; 120: 1454-1462

Die Diagnostik thromboembolischer Ereignisse (VTE) in der Schwangerschaft und Postpartalperiode fordert heraus, denn klinische Symptome wie Atemnot und Schwellung der Beine überlappen sich mit pathophysiologischen Prozessen in der Schwangerschaft. Gleichzeitig stellen VTE eine Hauptursache für Todesfälle bei Schwangeren in hochentwickelten Ländern dar. Mit Daten des RIETE-Registers ermittelten Elgendy et al. klinische Charakteristiken, Symptome, Management und Ergebnisse von Frauen, bei denen schwanger oder postpartal ein VTE aufgetreten war.

Das RIETE-Register (Registro Informatizado Enfermedad Trombo Embólica) ist eine internationale, multizentrische Sammlung von Patienten mit objektivierten VTE. Von 2001-2019 traten bei 596 Frauen während der Schwangerschaft VTE auf. 245 waren im 1., $124 \mathrm{im} \mathrm{2.} \mathrm{und} 227 \mathrm{im}$ 3. Trimester betroffen. 532 erlitten in der Postpartalperiode ( $\leq 2$ Monate nach der Entbindung) ein VTE. Insgesamt 739 hatten eine Venenthrombose (DVT) und 380 eine Lungenembolie (PE) mit oder ohne DVT. In postparta- len Fällen war bei $40 \%$ eine Sectio erfolgt. In einer dritten Gruppe waren < 50-jährige Patientinnen mit nicht schwangerschaftsassoziierten VTE. Verglichen mit den Kontrollen hatten Schwangere und junge Mütter ein anderes klinisches Profil:

- Risikofaktoren wie Immobilität, Malignome und Reisen kamen seltener vor (19\% vs. $60 \%$ ),

- Komorbiditäten wie Rauchen oder Niereninsuffizienz lagen seltener vor,

- Thrombophilien waren häufiger (53,2\% vs. $46 \%$ ) und

- $92 \%$ erhielten in der Schwangerschaft und $86 \%$ postpartal niedermolekulares Heparin.

PE kamen vor der Entbindung seltener vor als danach ( $27 \%$ vs. $42 \%$ ). Schwangere hatten außerdem anatomisch bedingt häufiger linksseitige (68\% vs. $51 \%$ ) sowie proximale DVT (78\% vs. $71 \%$ ) und erhielten öfter einen Vena-Cava-Filter (6\% vs. 4,2\%).

Von allen Frauen lagen Daten aus 3 Monaten und von $91 \%$ aus 180 Tagen nach dem VTE vor. Die Inzidenz der Komplikationen war gering. Die Rate rezidivierter DVT und
PE lag unter $1 \%$. Blutungen waren ebenfalls selten. Zwei Frauen starben an einer PE im Alter von 19 und 29 Jahren, 2 weitere Patientinnen mit postpartaler DVT starben im Zusammenhang mit einer Tumorerkrankung.

\section{FAZIT}

In der Schwangerschaft führten die gesteigerte Produktion von Gerinnungsfaktoren, die verminderte Verfügbarkeit von Protein S und die reduzierte Fibrinolyse zu einem hyperkoagulatorischen Status. Die häufigeren Thrombophilien sollten vor diesem Hintergrund eingeordnet werden, so die Autoren. Die Gesamtinzidenz von VTE in der Schwangerschaft und postpartal sei mit $0,025-1 \%$ gering. Wegen der überlappenden Symptome und eingeschränkten diagnostischen Möglichkeiten müsse die Differenzialdiagnose VTE aber immer erwogen werden.

Dr. med. Susanne Krome, Melle 